\title{
Trastuzumab-DM1: A Review of the Novel Immuno-Conjugate for HER2- Overexpressing Breast Cancer
}

\author{
Myra F. Barginear ${ }^{*}, 1$ and Daniel R. Budman ${ }^{2}$ \\ ${ }^{I}$ The Mount Sinai School of Medicine, The Breast Cancer Medicine Program at the Tisch Cancer Institute, One Gustave \\ L. Levy Place, Box 1079, New York, NY 10029, USA \\ ${ }^{2}$ New York University School of Medicine, Don Monti Division of Oncology, Monter Cancer Center of North Shore \\ University Hospital, USA
}

\begin{abstract}
Trastuzumab is a monoclonal antibody targeted against the HER2 tyrosine kinase receptor. Although trastuzumab is a very active agent in HER2-expressing breast cancer, the majority of patients with metastatic HER2 overexpressing breast cancer who initially respond to trastuzumab develop resistance within one year of initiation of treatment, and in the adjuvant setting, progress despite trastuzumab-based therapy. The immuno-conjugate TrastuzumabDM1 (T-DM1) was designed to combine the biological activity of trastuzumab with the targeted delivery of a highly potent antimicrotubule agent, DM1, a maytansine derivative, to HER2-expressing breast cancer cells. T-DM1 is the first immuno-conjugate with a non-reducible thioether linker in clinical trials. Here, we summarize evidence from pre-clinical studies, analyze clinical data, and discuss the potential clinical implications of T-DM1.
\end{abstract}

\section{INTRODUCTION}

Several methods to enhance and improve the selectivity of cytotoxic drugs, and thus significantly improve the therapeutic index, are currently being pursued. One particular method is targeting drug carriers such as antibodies; this is the objective of immuno-conjugates, in which cytotoxic drugs are attached via chemical linkers to antibodies that recognize cancer cell antigens and deliver the cytotoxic drug only to the cells of interest.

The therapeutic concept of drug targeting was founded on Paul Ehrlich's vision of "the magic bullet" [1]. As such, he predicted the emergence of targeted agents which have lessened effects on host tissue. However, serotherapy was limited by the lack of appropriate targets, the inability to make discrete antibodies with high specificity in large quantities, and the reproducibility of the characteristics of the poly-antisera. In the late 1970's, George Köhler and César Milstein fundamentally changed Immunology by developing the technology of hybridomas, the production of a desired specific antibody in large amounts [2]. These investigators showed that antibody-producing cells, of any given specificity, could be fused with a myeloma cell line and create an unlimited amount of monoclonal antibodies carrying that specificity [3]. This finding has led researchers, clinicians, and especially biotechnology companies to focus on new diagnostic and therapeutic agents.

Lessons from the development of immunoconjugates can be learned from the biodistribution of immunoconjugates and the relationship between toxicity and efficacy. This is observed by the success of Yttrium-90 $(90 \mathrm{Y})$-ibritumomab

*Address correspondence to this author at the Tisch Cancer Institute, The Mount Sinai School of Medicine, One Gustave L. Levy Place, Box 1079, New York, NY 10029, USA; Tel: (212) 241-3650; Fax: (212) 876-5276;

E-mail: Myra.Barginear@mssm.edu tiuxetan (Zevalin ${ }^{\circledR}$, Biogen) and Iodine-131 (131I)tositumomab (Bexxar ${ }^{\circledR}$, Glaxo SmithKline) $[4,5]$. Both of these radio-immunoconjugate therapies target the $\mathrm{CD} 20$ antigen that is expressed within normal pre-B lymphocytes but not on stem cells or plasma cells. The anatomic distribution of CD20+ cells on both normal and malignant lymphocytes within blood and bone marrow may influence the activity and toxicity of radio-immunoconjugates.

Trastuzumab is a monoclonal antibody targeted against the HER2 tyrosine kinase receptor. Although trastuzumab is a very active agent in HER2-expressing breast cancer, the majority of patients with metastatic HER2 overexpressing breast cancer who initially respond to trastuzumab develop resistance within one year of initiation of treatment, and in the adjuvant setting, progress despite trastuzumab-based therapy [6-9]. The immuno-conjugate Trastuzumab-DM1 (TDM1) was designed to combine the biological activity of trastuzumab (Herceptin ${ }^{\circledR}$, Genentech) with the targeted delivery of a highly potent antimicrotubule agent, DM1 (ImmunoGen), a maytansine derivative, to human epidermal growth factor receptor type 2 (HER2)-expressing breast cancer cells. The aim of immuno-conjugates such as T-DM1 is to target intracellular delivery of both drugs with high specificity to avoid normal tissues.

The anti-mitotic drug maytansine was chosen for use in the targeted delivery approach because of its high in vitro potency $[10,11]$. It was originally isolated from members of the plant families Rhamnaceae and Euphorbiaceae, as well as some mosses [12]. The maytansinoids bind tubulin competitively with vinca alkaloids and are approximately 100 times more potent than vincristine [12-14]. To link maytansinoids to antibodies through disulfide bonds, a thiolcontaining maytansinoid, DM1 (N-methyl-N-[3-mercapto-1oxopropyl]-L-alanine ester of maytansinol), an analog of the clinically-studied drug maytansine) was synthesized. In the case of T-DM1, DM1 is linked to trastuzumab using the 
bifunctional reagent, SMCC ( $N$-succinimidyl-4(maleimidomethyl) cyclohexanecarboxylate.) SMCC is first added to lysine residues on the protein to produce a linker modified antibody. The thiol group in DM1 is then reacted with the maleimide group of the linker to form the nonreducible thioether bond (Fig. 1). Each antibody contains an average of 3.5 drugs, however the product contains a distribution from 0 to 8 drugs per antibody [15]. T-DM1 is the first immuno-conjugate with a nonreducible thioether linker in clinical trials.

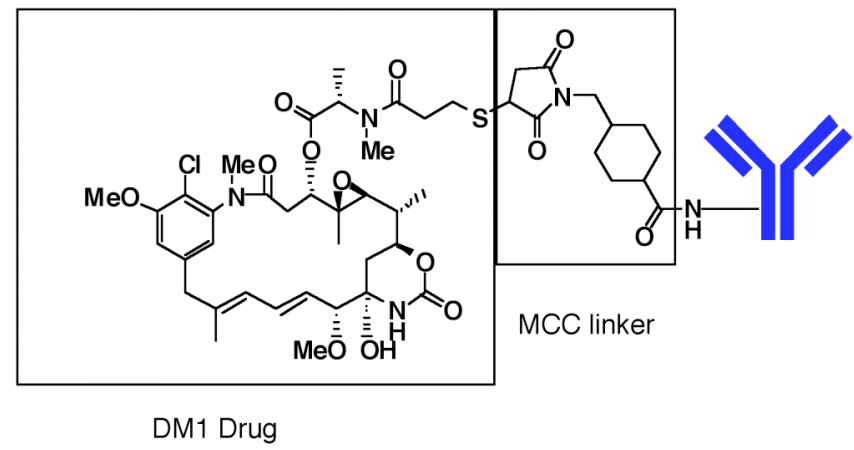

Fig. (1). Illustration of T-DM1. The average number of DM1 molecules per monoclonal antibody is 3.5 [45].

A review of the literature regarding T-DM1, its safety, efficacy, and toxicities was conducted by searches on Medline, Embase, Web of Science, and scientific meeting proceedings. Here, we summarize evidence from pre-clinical studies, analyze clinical data, and discuss the potential clinical implications of T-DM1.

\section{HER2 POSITIVE BREAST CANCER}

Overexpression of HER2, also referred to as HER2/neu or ErbB-2, was first described more than two decades ago $[16,17]$. HER2 overexpression or gene amplification is observed in approximately 20 to $30 \%$ of invasive breast cancers. HER2 overexpression has been reported to be a poor prognostic factor and a predictive risk factor for decreased disease-free survival and overall survival in patients with breast cancer $[18,19]$. Consequently, therapies which target HER2 are attractive in treating HER2overexpressing breast cancer. One such therapy is trastuzumab, a humanized monoclonal immunoglobulin (IgG) that targets the extracellular domain of the HER2 receptor. Trastuzumab was approved in 1998 for the treatment of metatastic breast cancer and in 2006 for the adjuvant treatment of HER2 overexpressing breast cancer [20].

The exact mechanisms in which trastuzumab causes regression of HER2-overexpressing tumors are still being clarified, but several effects have been reported in the literature [21]. They include antibody-dependent cellular cytotoxicity, angiogenesis inhibition, and reduced downstream signaling through phosphatidylinositol 3-kinase and mitogen-activated protein kinase, ultimately resulting in cell-cycle arrest and apoptosis [21-25].

\section{Trastuzumab Resistance}

Although the selection of trastuzumab is based on the presence of HER2 overexpression, the simple presence of HER2 is not sufficient to ensure that trastuzumab will be effective in first-line treatment or guarantee that resistance will not develop.

The objective response rates for patients with HER2 overexpressing tumors to single agent trastuzumab range from $12 \%$ to $34 \%$, for a median duration of 9 months $[7,8$, $26,27]$. The median time to progression in patients who have not received prior chemotherapy and who have received prior chemotherapy for HER2 overexpressing metastatic breast cancer, is 4.9 months and 7.4 , respectively [7, 8]. Thus, patients who respond to trastuzumab develop resistance within one year.

In the adjuvant setting, the administration of trastuzumab in combination with or following chemotherapy improves the disease-free and overall survival rates in patients with early stage breast cancer [6,9]. However, approximately $15 \%$ of these women still develop metastatic disease despite trastuzumab-based adjuvant chemotherapy. Understanding the mechanisms that contribute to trastuzumab resistance and identifying novel agents is critical to improving the survival of patients whose tumors overexpress HER2.

\section{Trastuzumab - DM1}

A normal breast cell may have 20,000 HER2 receptors, whereas a breast cancer cell may express as many as 1.5 million copies per cell, which makes HER2 a potential target for immuno-conjugate therapy [28]. Preclinical and clinical studies indicate that trastuzumab combines extremely well with microtubule-directed agents [29, 30]. Thus, it was of particular interest to conjugate trastuzumab to the extremely potent drug maytansine.

The immuno-conjugates in development have been reported that use either antibody lysines or cysteines as the sites of drug attachment (31-34). Immuno-conjugates containing DM1 have previously been reported using several targeting antibodies [13-35]. In these studies, the linker attaching the drug to the protein lysines used a partially hindered, but still reducible disulfide. Trastuzumab linked to DM1 through a nonreducible thioether linker (SMCC) was compared to versions using disulfide linkers and appeared to offer improvements to both safety and efficacy [37]. Although the non-reducible linker is significantly more stable in circulation than the disulfide, it can still release active DM1 following internalization into tumor cells [36]. Work by Erickson, et al. [38] suggests that the immunoconjugate is proteolytically degraded to release lysine-MCCDM1, which is still a potent anti-mitotic agent.

\section{TRASTUZUMAB-DM1 CLINICAL TRIALS}

\section{Pre-Clinical}

Lewis-Phillips and colleagues reported the pharmacokinetics, efficacy, and toxicity of T-DM1[37]. The biological effects of disulfide linkers were investigated by constructing various T-DM1 conjugates; the stability of the linker not only compromises potency but can also exacerbate toxicity. Trastuzumab conjugated to DM1 through a nonreducible thioether linkage displayed better efficacy and lower toxicity than trastuzumab conjugated with reducible disulfide linkers. This was also confirmed by Kovtan and colleagues [38]. T-DM1 is internalized upon binding to HER2-overexpressing tumor cells and it is believed that 
maytansine is released secondary to intracellular proteolytic degradation.

The antiproliferative effects of T-DM1 were evaluated in normal (MCF 10A breast epithelial cells) and tumor (breast carcinoma BT-474, SK-BR-3, MCF7, MDA-MB-468, MDAMB- 361, HCC1954, lung carcinoma Calu 3, and ovarian carcinoma line SK-OV-3) cell lines. HER2overexpressing, trastuzumab-resistant tumor cells responded to T-DM1 whereas cell lines without overexpression were unaffected. Cell cycle analysis of conjugate-treated cells revealed cell cycle arrest in the $\mathrm{G}_{2}-\mathrm{M}$ phase.

In vivo efficacy was determined in HER2-overexpressing breast cancer mouse models. Complete tumor regression for the duration of the study (126 days) was observed in mice receiving T-DM1. In contrast, unconjugated trastuzumab induced an initial decrease in tumor size, followed by regrowth upon cessation of treatment. In addition, T-DM1 was active in HER2-overexpressing, trastuzumab-refractory tumors [37].

Junttila and colleagues recently reported that trastuzumab conjugated to DM1 did not affect the mechanism of action of traztuzumab [39]. Cell lines overexpressing HER2 were used to assess in vitro efficacy and a trastuzumabinsensitive, HER2-transgenic mouse model was used to assess in vivo efficacy. The affinity of T-DM1 for HER2 was nearly identical to trastuzumab. Drug conjugation did not adversely affect trastuzumab's inhibition of HER2 shedding or downregulation of AKT signaling. Notably, T-DM1 was active against mouse models that were insensitive to either trastuzumab or lapatinib (Tykerb ${ }^{\circledR}$, GlaxoSmithKline). These results, along with the favorable efficacy and safety profile of trastuzumab linked to DM1 through a non-reducible linker, have advanced T-DM1 into clinical development.

\section{Clinical}

Two phase I studies have been conducted with T-DM1, administered weekly or every 3 weeks, in patients with advanced HER2 overexpressing breast cancer [40, 41]. Beeram et al. reported six dose levels of intravenous TDM1, scheduled every three weeks, in twenty-four HER2 positive breast cancer patients [40]. Patients had progressed on at least one trastuzmab-containing regimen with the median number of 92 weeks of prior trastuzumab therapy. Patients with significant cardiac disease, who had received prior doses of anthracyclines, and/or Grade 2 neuropathy, were excluded. The median age was 51 and all patients had an ECOG performance status of 0 or 1 (Table 1) [40].

The six dose levels of T-DM1 were administered: 0.3 $\mathrm{mg} / \mathrm{kg}(\mathrm{n}=3), 0.6 \mathrm{mg} / \mathrm{kg}(\mathrm{n}=1), 1.2 \mathrm{mg} / \mathrm{kg} \quad(\mathrm{n}=1), 2.4 \mathrm{mg} / \mathrm{kg}$ $(\mathrm{n}=1), 3.6 \mathrm{mg} / \mathrm{kg}(\mathrm{n}=15)$, and $4.8 \mathrm{mg} / \mathrm{kg}(\mathrm{n}=3)$. Side effects included grade 1 and 2 transaminase elevations, thrombocytopenia, fatigue and nausea. Constipation, anemia, and neuropathy were also reported. Grade 3 and 4 thrombocytopenia was reported in three patients of whom two had Grade 4 events at $4.8 \mathrm{mg} / \mathrm{kg}$, the dose limiting toxicity. One patient experienced Grade 3 neutropenia and another pulmonary hypertension. No cardiac events were reported.

The median progression-free survival at $3.6 \mathrm{mg} / \mathrm{kg}$, the maximum tolerated dose, was 9.8 months. Additionally, thrombocytopenia, at the maximum tolerated does, was reported to be grade 1, rapidly reversible, and not cumulative. Partial responses were observed in 6/16 patients at $2.4 \mathrm{mg} / \mathrm{kg}(\mathrm{n}=1)$ and $3.6 \mathrm{mg} / \mathrm{kg}(\mathrm{n}=5)$. These patients had prior treatment with trastuzumab and other tublin-binding cytotoxic agents such as paclitaxel, docetaxel, and vinorelbine. An additional 9 patients were reported to have stable disease.

In the second phase I trial, Holden and colleagues reported weekly dosing of T-DM1 in fifteen patients with advanced HER2-positive breast cancer [41]. Patients had progressed on at least one trastuzmab-containing regimen with the median number of 122 weeks of prior trastuzumab therapy and an average of two prior chemotherapy regimens. The median age was 53 and all patients had an ECOG performance status of 0 or 1 . T-DM1 was dosed at $1.2 \mathrm{mg} / \mathrm{kg}$ $(\mathrm{n}=3), 1.6 \mathrm{mg} / \mathrm{kg}(\mathrm{n}=3), 2.0 \mathrm{mg} / \mathrm{kg}(\mathrm{n}=3), 2.4 \mathrm{mg} / \mathrm{kg} \quad(\mathrm{n}=7)$, and $2.9 \mathrm{mg} / \mathrm{kg}(\mathrm{n}=3)$ intravenously weekly.

Burris and colleagues initially reported phase II data of T-DM1 in HER2-positive metastatic breast cancer at the American Society of Clinical Oncology Breast Cancer Symposium and updated the results at the San Antonio Breast Cancer Symposium, December 2008 [42-44]. Enrollment completed with 112 patients. Patients were eligible if they had progression of disease on or within sixty days of receiving HER2 directed therapy and no active brain metastases, defined as symptomatic or requiring treatment within the last three months. Additional eligibility criteria included prior treatment with one or more chemotherapy regimens and no history of significant cardiac disease. The median age was 55 (33-79). The majority of patients had an ECOG performance status of 0 or $1,68 \%$ had more than three metastatic sites, primarily liver and lung, and $8 \%$ had brain metastases. The median number of prior cytotoxic therapies was three (1-12) with $68 \%$ receiving an anthracycline. The median duration of prior traztuzumab was 77 weeks. Fifty-five percent of patients had received prior lapatinib therapy with a median duration of prior lapatinib therapy for 26 weeks.

Patients were treated with T-DM1 dosed at $3.6 \mathrm{mg} / \mathrm{kg}$ intravenously every three weeks and assessed every two cycles for toxicity and efficacy. Echocardiograms or MUGA scans were done after cycle 1 and cycle 2 and every other cycle thereafter. The average number of cycles administered was five; three patients were dose reduced to $3 \mathrm{mg} / \mathrm{kg}$ every three weeks. Pharamacokinetic parameters indicated the average terminal half-life was 89 hours and peak serum levels were less than $10 \mathrm{ng} / \mathrm{ml}$. The preliminary pharmacokinetic analyses were similar to those reported for the maximum tolerated does in the phase I study by Beerman and colleagues [45].

The most common adverse advents were fatigue, nausea, and headache. Grade 3 and 4 adverse events included thrombocytopenia $(n=8)$, hypokalemia $(n=6)$, fatigue $(n=4)$, infection $(n=3)$, musculoskeletal chest pain $(n=2)$, convulsions $(n=2)$, thromboses $(n=2)$, dyspnea $(n=3)$, pleural effusion $(n=3)$, and epistaxis $(n=2)$. There were no Grade 3 or 4 cardiac dysfunction reported.

Fifty-six of the 112 patients have discontinued the study. Forty-four patients have discontinued the study secondary to 
Table 1. Clinical Data Available, to Date, for T-DM1 in Patients with Metastatic HER2 Overexpressed Breast Cancer on Previous HER2 Directed Therapy

\begin{tabular}{|c|c|c|c|c|c|c|c|c|c|c|}
\hline & Phase & $\mathbf{N}$ & $\begin{array}{l}\text { Media } \\
\text { n Age }\end{array}$ & $\begin{array}{l}\text { Median \# } \\
\text { Prior T } \\
\text { (Weeks) }\end{array}$ & Dose Levels & SD & PR & CR & Grade 3 or $4 \mathrm{AE}$ & Conclusion \\
\hline Beerman et al & I & 24 & 51 & 91.6 & $\begin{array}{c}.3-4.8 \\
\mathrm{mg} / \mathrm{kg} \text { every } \\
3 \text { weeks }\end{array}$ & $5 / 16$ & $6 / 16$ & $6 / 16$ & $\begin{array}{c}\text { Thrombocytopenia } \\
\text { Neutropenia }\end{array}$ & $\begin{array}{c}\text { MTD: } 3.6 \mathrm{mg} / \mathrm{kg} \text { every } 3 \\
\text { weeks }\end{array}$ \\
\hline Holden et al & I & 28 & 53 & 122 & $\begin{array}{l}1.2-2 \mathrm{mg} / \mathrm{kg} \\
\text { weekly }\end{array}$ & NR & $15 / 26$ & NR & Thrombocytopenia & $\begin{array}{c}\text { MTD: } 2.4 \mathrm{mg} / \mathrm{kg} \text { every } 3 \\
\text { weeks }\end{array}$ \\
\hline Burris et al & II & 11 & 55 & 76.6 & $\begin{array}{l}3.6 \mathrm{mg} / \mathrm{kg} \\
\text { every } 3 \\
\text { weeks }\end{array}$ & $12 / 30$ & $12 / 30$ & $1 / 30$ & $\begin{array}{c}\text { Thrombocytopenia } \\
\text { Hypokalemia } \\
\text { Fatigue } \\
\text { Infection } \\
\text { Musculoskeletal } \\
\text { chest pain } \\
\text { Convulsions } \\
\text { Thromboses } \\
\text { Dyspnea } \\
\text { Pleural effusions } \\
\text { Epistaxis }\end{array}$ & $\begin{array}{l}\text { Antitumor activity in } \\
\text { trastuzumab and lapatinib } \\
\text { pre-treated pts } \\
\text { Single agent activity in } \\
\text { Her2-positive breast } \\
\text { cancer } \\
\text { Phase III in } 2009\end{array}$ \\
\hline
\end{tabular}

T: trastuzumab, N: number, SD: stable disease, PR: partial response, CR: complete response, AE: adverse event, NR: not reported, MTD: maximum tolerated dose.

progression of disease, six secondary to patient or physician's decision to discontinue the study, five secondary to adverse events unrelated to T-DM1, and one death on the study secondary to progression of disease.

The primary endpoint was overall response rate; the objective overall response rate was 39.3\% (95\% confidence interval $(\mathrm{CI})=30-49)$, and the confirmed overall response rate, repeat imaging done on two occasions more than four weeks apart, was $27 \%(95 \% \mathrm{CI}=19.4-36.1)$. Of the 62 patients exposed to prior lapatinib therapy, sixty were evaluable for efficacy. Objective responses were observed in 23/60 (38.30\%, 95\% CI=19.4-36.1).

In October 2008, Genentech indicated that they plan to initiate a Phase III, randomized, multicenter, international trial designed to compare the safety and efficacy of T-DM1 with that of capecitabine (Xeloda ${ }^{\circledR}$, Roche) and lapatinib in patients with metastatic breast cancer that overexpress HER2. A total of 580 patients will be enrolled at approximately 260 sites worldwide. The company has also announced that they have initiated a Phase II trial comparing T-DM1, given as a single agent, to trastuzumab plus docetaxel as a first-line treatment in metastatic HER2positive breast cancer. Currently, Genentech is enrolling a multicenter, open-label extension study. Patients who received T-DM1 in a Genentech-sponsored study and completed T-DM1 or who continue to receive T-DM1 at the time of the parent study closure are eligible for continued treatment. Patients can receive T-DM1 until disease progression or unacceptable toxicity for as long as the extension study remains open. More information on these trials can be found at www.clinicaltrials.gov.

\section{DISCUSSION}

The immono-conjugate T-DM1 has displayed significant in vitro and in vivo potency. Patients enrolled in the Phase I and II trials were heavily pretreated with cytotoxic therapy as well as HER2-targeted therapy. The median duration of prior traztuzumab or lapatinib therapy was over 1 year [43]. This finding establishes that HER2 remains a viable target, in HER2 overexpresing breast cancer, even after trastuzumab is ineffective.

There is clinical evidence that continued treatment with trastuzumab beyond progression improves activity of subsequent chemotherapy in patients with HER2-positive breast cancer [46]. Trastuzumab can induce an immunemediated, target-cell killing by antibody-dependent, cellmediated cytotoxicity $[47,48]$. It is hypothesized that, under the selective pressure of continuous antibody exposure, breast cancer cells may be able to bypass the antiproliferative action of trastuzumab but leave a chemotherapy-sensitizing mechanism intact [46]. The reintroduction or continuation of trastuzumab via T-DM1 may be very effective in patients with metastatic breast cancer who are heavily pretreated.

The delivery of the active cytotoxic agent, maytansine, through the drug delivery system of trastuzumab is significant. The linker chemistry is of upmost importance with regards to efficacy and tolerability. The thio-ether linked T-DM1 increases serum stability relative to a disulfide linker version (37) and appears to undergo endosomal degradation (17), resulting in intracellular release of the cytotoxic agent (17), thereby improving efficacy and tolerability. 
T-DM1 is also well tolerated. Grade 3 and 4 thrombocytopenia and elevated transaminases were reported to be reversible [45]. Notably, there were no Grade 3 or 4 cardiac dysfunction reported, and nearly $40 \%$ of patients enrolled in the Phase II trial had received a prior anthracycline. Cardiotoxicity remains a major concern withthe use of trastuzumab. The association between impairment of the left cardiac ventricle and trastuzumab was first observed in one of the early prospective trastuzumab trials; cardiotoxicity was seen in $27 \%$ of patients treated with trastuzumab and anthracyclines, concurrently, in $13 \%$ of patients treated with trastuzumab and paclitaxel, and in 5\% of patients treated with trastuzumab alone [8].

In addition, T-DM1's antitumor results are extremely note-worthy in a heavily pretreated patient population. The overall response rates were observed in over $38 \%$ of patients. These developments provide us with optimism for the future, but further investigation of T-DM1 will need to focus on the mechanisms of resistance and toxicity, so that T-DM1 can be integrated into treatment schemes safely. The use of T-DM1 with other immuno-conjugates, which could possibly provide non-cross-resistant therapeutic effects without cumulative toxicities, should also be explored [49].

\section{FUTURE DIRECTIONS}

In the past few decades we have seen significant progress in the development of immuno-conjugates as anti-cancer agents. Improving the therapeutic index of these drugs is imperative and will probably result from targeting antigens and increasing linker stability, as demonstrated by T-DM1. The progress made in the targeted delivery approach with TDM1 as well as the clinical and pre-clinical results have been very promising. It is plausible that T-DM1 may be a treatment strategy for patients with HER2 overexpressed breast cancer that progress on first line HER2- directed therapy. The Phase III data results for T-DM1 are anxiously awaited.

\section{REFERENCES}

[1] Lokaj J, John C. [ilya ilich metchnikov and paul ehrlich: 1908 nobel prize winners for their research on immunity]. Epidemiol Mikrobiol Imunol 2008; 57: 119-24.

[2] Funaro A, Horenstein AL, Santoro P, et al. Monoclonal antibodies and therapy of human cancers. Biotechnol Adv 2000; 18: 385-401.

[3] Uhr JW. The 1984 Nobel Prize in medicine. Science 1984; 226: 1025-28.

[4] Kaminski MS, Tuck M, Estes J, et al. 131i-tositumomab therapy as initial treatment for follicular lymphoma. N Engl J Med 2005; 352: 441-49.

[5] Jacobs SA, Vidnovic N, Joyce J, et al. Full-dose 90y ibritumomab tiuxetan therapy is safe in patients with prior myeloablative chemotherapy. Clin Cancer Res 2005; 11: 7146s-50s.

[6] Piccart-Gebhart MJ, Procter M, Leyland-Jones B, et al. Trastuzumab after adjuvant chemotherapy in her2-positive breast cancer. N Engl J Med 2005; 353: 1659-72.

[7] Seidman AD, Fornier MN, Esteva FJ, et al. Weekly trastuzumab and paclitaxel therapy for metastatic breast cancer with analysis of efficacy by her2 immunophenotype and gene amplification. J Clin Oncol 2001; 19: 2587-95.

[8] Slamon DJ, Leyland-Jones B, Shak S, et al. Use of chemotherapy plus a monoclonal antibody against her2 for metastatic breast cancer that overexpresses her2. N Engl J Med 2001; 344: 783-92.
[9] Romond EH, Perez EA, Bryant J, et al. Trastuzumab plus adjuvant chemotherapy for operable her2-positive breast cancer. N Engl J Med 2005; 353: 1673-84.

[10] Higashide E, Asai M, Ootsu K, et al. Ansamitocin, a group of novel maytansinoid antibiotics with antitumour properties from nocardia. Nature 1977; 270: 721-22.

[11] Cassady JM, Chan KK, Floss HG, et al. Recent developments in the maytansinoid antitumor agents. Chem Pharm Bull (Tokyo) 2004; 52: 1-26.

[12] Yu TW, Bai L, Clade D, et al. The biosynthetic gene cluster of the maytansinoid antitumor agent ansamitocin from actinosynnema pretiosum. Proc Natl Acad Sci U S A 2002; 99: 7968-73.

[13] Tassone P, Goldmacher VS, Neri P, et al. Cytotoxic activity of the maytansinoid immunoconjugate b-b4-dm1 against cd138+ multiple myeloma cells. Blood 2004; 104: 3688-96.

[14] Wang L, Amphlett G, Blattler WA, et al. Structural characterization of the maytansinoid-monoclonal antibody immunoconjugate, hun901-dm1, by mass spectrometry. Protein Sci 2005;14:2436-2446.

[15] Blättler WA, Chari RVJ, Vite GD, Altmann KH, Eds. Anticancer Agents - Frontiers in Cancer Chemotherapy, American Chemical Society, Washington 2001; 317-38.

[16] Schechter AL, Stern DF, Vaidyanathan L, et al. An erb-b-related gene encoding a 185,000-mr tumour antigen. Nature 1984; 312: 513-16.

[17] Hudis CA. Trastuzumab--mechanism of action and use in clinical practice. N Engl J Med 2007; 357: 39-51.

[18] Pegram MD, Pauletti G, Slamon DJ. Her-2/neu as a predictive marker of response to breast cancer therapy. Breast Cancer Res Treat 1998; 52: 65-77.

[19] Slamon DJ, Clark GM, Wong SG, et al. Human breast cancer: Correlation of relapse and survival with amplification of the her2/neu oncogene. Science 1987; 235:177-82.

[20] Tokunaga E, Oki E, Nishida K, et al. Trastuzumab and breast cancer: Developments and current status. Int J Clin Oncol 2006; 11: 199-08.

[21] Nahta R, Esteva FJ. Her2 therapy: Molecular mechanisms of trastuzumab resistance. Breast Cancer Res 2006; 8: 215.

[22] Baselga J, Albanell J, Molina MA, et al. Mechanism of action of trastuzumab and scientific update. Semin Oncol 2001; 28: 4-11.

[23] Arnould L, Gelly M, Penault-Llorca F, et al. Trastuzumab-based treatment of her2-positive breast cancer: An antibody-dependent cellular cytotoxicity mechanism? Br J Cancer 2006; 94: 259-67.

[24] Izumi Y, Xu L, di Tomaso E, et al. Tumour biology: Herceptin acts as an anti-angiogenic cocktail. Nature 2002; 416: 279-80.

[25] Wen XF, Yang G, Mao W, et al. Her2 signaling modulates the equilibrium between pro- and antiangiogenic factors via distinct pathways: Implications for her2-targeted antibody therapy. Oncogene 2006; 25: 6986-96.

[26] Cobleigh MA, Vogel CL, Tripathy D, et al. Multinational study of the efficacy and safety of humanized anti-her2 monoclonal antibody in women who have her2-overexpressing metastatic breast cancer that has progressed after chemotherapy for metastatic disease. J Clin Oncol 1999; 17: 2639-48.

[27] Vogel CL, Cobleigh MA, Tripathy D, et al. Efficacy and safety of trastuzumab as a single agent in first-line treatment of her2overexpressing metastatic breast cancer. J Clin Oncol 2002; 20: 719-26.

[28] Carter P, Smith L, Ryan M. Identification and validation of cell surface antigens for antibody targeting in oncology. Endocr Relat Cancer 2004; 11: 659-87.

[29] Baselga J, Norton L, Albanell J, et al. Recombinant humanized anti-her2 antibody (herceptin) enhances the antitumor activity of paclitaxel and doxorubicin against her $2 /$ neu overexpressing human breast cancer xenografts. Cancer Res 1998; 58: 2825-31.

[30] Pegram M, Hsu S, Lewis G, et al. Inhibitory effects of combinations of her-2/neu antibody and chemotherapeutic agents used for treatment of human breast cancers. Oncogene 1999; 18: 2241-51.

[31] Carter PJ, Senter PD. Antibody-drug conjugates for cancer therapy. Cancer J 2008; 14: 154-69. 
[32] Lambert JM. Drug-conjugated monoclonal antibodies for the treatment of cancer. Curr Opin Pharmacol 2005; 5: 543-49.

[33] Damle NK. Antibody-drug conjugates ace the tolerability test. Nat Biotechnol 2008; 26: 884-5.

[34] Hamblett KJ, Senter PD, Chace DF, et al. Effects of drug loading on the antitumor activity of a monoclonal antibody drug conjugate. Clin Cancer Res 2004; 10: 7063-70.

[35] Smith SV. Technology evaluation, Hun901-dm1, immunogen. Curr Opin Mol Ther 2005; 7: 394-401.
[36] Kratz F, Abu Ajaj K, Warnecke A. Anticancer carrier-linked prodrugs in clinical trials. Expert Opin Investig Drugs 2007; 16 1037-58.

[37] Lewis Phillips GD, Li G, Dugger DL, et al. Targeting her2-positive breast cancer with trastuzumab-dm1, an antibody-cytotoxic drug conjugate. Cancer Res 2008; 68: 9280-90.

[38] Erickson HK, Park PU, Widdison WL, et al. AntibodyMaytansinoid Conjugates Are Activated in Targeted Cancer Cells by Lysosomal Degradation and Linker-Dependent Intracellular Processing. Cancer Res 2006; 66: 4426-33.

(C) Barginear and Budman; Licensee Bentham Open .

This is an open access article licensed under the terms of the Creative Commons Attribution Non-Commercial License (http://creativecommons.org/licenses/by-nc/ 3.0/) which permits unrestricted, non-commercial use, distribution and reproduction in any medium, provided the work is properly cited. 